\title{
Dopant Impurity Induced Nanofaceting on Silicon Nanowire Sidewalls
}

\author{
F. Li, P.D. Nellist, C. Lang, and D.J.H. Cockayne \\ Department of Materials, University of Oxford, Parks Road, Oxford, OX1 3PH, UK
}

Being one of the most important semiconductor nanostructures, silicon nanowires (SiNWs) have stimulated great interest because of their scientific importance and potential applications as the building blocks for future nanotechnologies as functional devices, including transistor components [1], chemical/biological sensors [2], and nanoelectromechanical systems [3]. For example, SiNWs with axially varying doping to generate junctions could be used as the channel in nanoscale fieldeffect transistors [4]. An important issue for functional devices based on nanowire structures is the control of the wire surface, because the surface plays an important role in controlling nanowire properties due to the high surface area to volume ratio. One important issue is the dependence of surface properties of SiNWs on impurity doping and the mechanisms that drive the nanowire growth.

In this work, axially-doped $p$-type/n-type/intrinsic ( $p$ - $n-i)$ SiNWs were grown by the vapor-liquidsolid approach using gold as the catalyst. The nanowire sidewalls exhibit periodic nanofaceting, which is strongly dopant-dependent, i.e., sidewall facets occur in the boron-doped ( $p$-type) region, while both the phosphorus-doped (n-type) and the intrinsic SiNW regions exhibit smooth surface morphology (FIG. 1). High resolution transmission electron microscopy (HRTEM) images show that the nanowires are axially oriented along $\langle 111\rangle$, and the crystallographic planes on which the nanofaceting of the sidewalls occurs are $\{111\}$ and $\{100\}$ (FIG. 2(a)). The nanofaceting occurs when the diborane dopant gas is introduced; this arises because the diborane dopant gas alters the nanowire growth mechanism and behavior by enhancing the sidewall growth and creating a faceted sidewall surface [5].

Based on these experimental observations, a thermodynamic model predicting the nanofacet period has been developed for a nanowire structure. In this model, an extra energy cost term arising from the formation of apexes between facets is considered, and the facet size is predicted to decrease as the wire diameter increases [6]. This model consistently explains the experimentally observed dependence of facet period on wire diameter in the boron-dopant induced nanofaceting on SiNW sidewalls (FIG. 2(b)).

References

[1] Y. Cui and C. M. Lieber, Science 291 (2001) 851.

[2] Z. Li et al., Nano Lett. 4 (2004) 245.

[3] R. He and P. Yang, Nat. Nanotechnol. 1 (2006) 42.

[4] M.S. Gudiksen et al., Nature (London) 415 (2002) 617.

[5] F. Li et al., Appl. Phys. Lett. 94 (2009) 263111.

[6] F. Li et al., ACS Nano (2010) in press. 


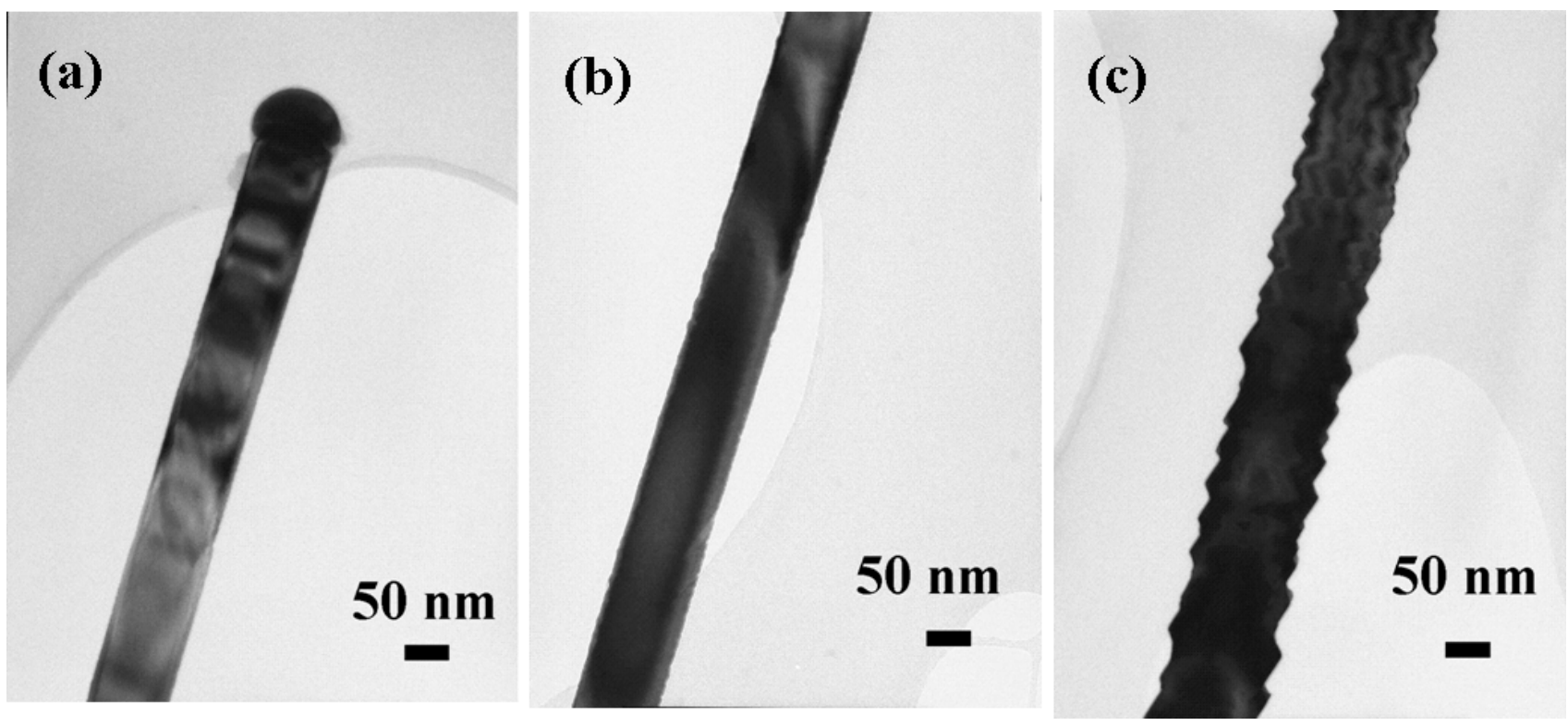

FIG. 1. TEM images of an axial heterostructured $p-n-i$ SiNW taken along the wire growth direction. (a) The intrinsic SiNW region; (b) the P-doped ( $n$-type) SiNW region; and (c) the B-doped ( $p$-type) SiNW region. (All are viewed from the $<110>$ direction.)

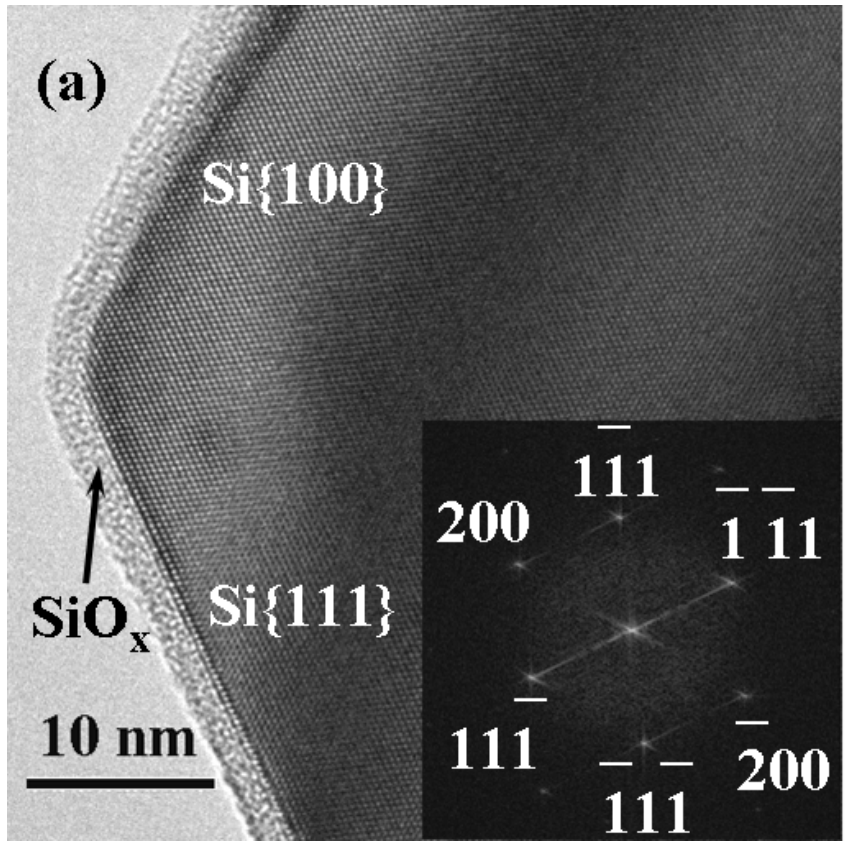

(b)

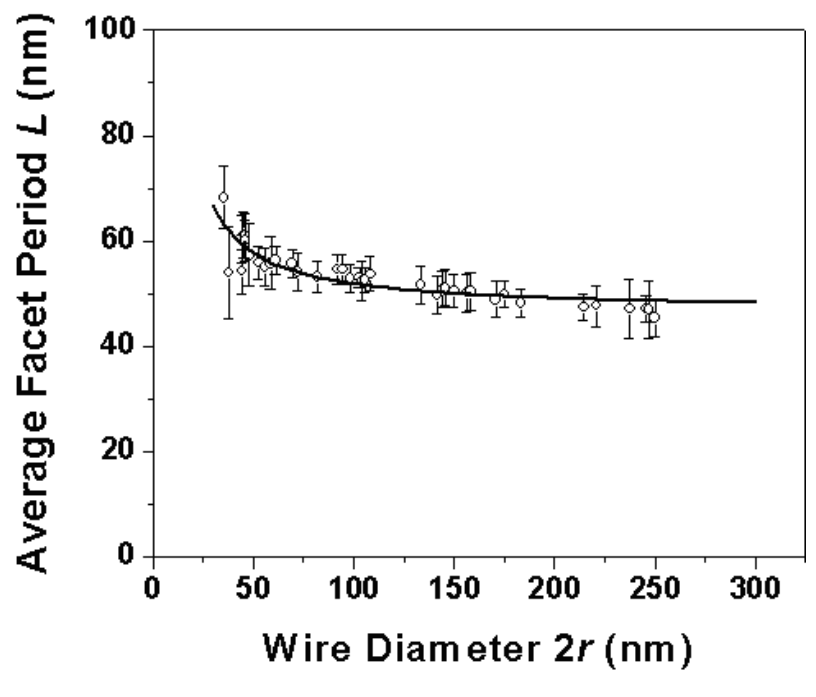

FIG. 2. (a) HRTEM image of the nanofacets on SiNW sidewalls, inset: Fourier transform of the image; and (b) the dependence of average facet period on the wire diameter of the $<111>$-oriented axial heterostructured SiNWs in the faceted B-doped region. The solid line through the data points is the nonlinear least-squares fit to the model. 